\title{
St-qR pattern: new sign for diagnosis of anterior myocardial infarction during right ventricular pacing
}

\author{
Agustin Castellanos, Jr., Robert Zoble, Pat M. Procacci, Robert J. Myerburg, and Barouh V. \\ Berkovits \\ From the Medical Service, Veterans Administration Hospital, and the Department of Medicine, Division of \\ Cardiology, University of Miami School of Medicine, Miami, Florida, U.S.A.
}

In 4 patients with recent anterior wall myocardial infarction involving the ventricular septum, bipolar right ventricular apical stimulation resulted in small negative deflections preceding larger $R$ waves (St-qR pattern) in leads I, aVL, and (except for Case 3) V6.

Abnormal $S T$ segment elevation after St-qR pattern was noted in only 2 cases. The presence or absence of repolarization changes probably reflected the stage of infarction during which electrocardiograms were obtained.

The mechanisms of these changes are similar to those responsible for the $q R$ morphology in left bundlebranch block complicated by septal infarction, as shown by the case in which both patterns were seen during spontaneous left bundle-branch block as well as during right ventricular stimulation.

The St-qR pattern may not be recorded when the ventricular complexes are distorted by large spikes (as when unipolar pacing is used) or when stimulation is performed from the upper portion of the right septal surface, an area to which the infarction does not extend.

In tracings showing sinus rhythm and left bundlebranch block small q waves in leads I, aVL, V5, and V6 have been attributed to coexisting anteroseptal wall myocardial infarction (Sodi-Pallares, Rodriquez, and Bisteni, 1952; Sodi-Pallares et al., 1963; Massie and Walsh, 1960). The resemblance between QRS complexes produced by right ventricular endocardial stimulation through catheter electrodes and those considered to be characteristic of true left bundle-branch block suggested to Cardenas et al. (1972) that the criteria used to diagnose myocardial infarction in the presence of left bundle-branch block could also be applied to pacemaker-induced QRS complexes.

The present communication corroborates the work of Cardenas et al. and also stresses why their assumptions have not received sufficient attention.

\section{Case reports}

Twelve-lead electrocardiograms were obtained from 4 patients with acute anterior wall myocardial infarction in which bipolar electrical stimulation had to be used for symptomatic AV block, presumably due to intra-Hisian lesions in Case I and to bilateral bundle-branch block in the remaining 3 patients. During sinus rhythm the Received I9 April 1973. tracings showed anteroseptal myocardial infarction in 3 (Case I, Fig. I; Case 2, Fig. 3; and Case 3, Fig. 5) and extensive anterior (anteroseptal and high lateral) myocardial infarction in the remaining one (Fig. 7).

Bipolar right ventricular apical pacing resulted in a superior and leftward axis shift with a predominant $S$ wave in right chest leads $V_{I}$ and $V_{2}$ in all cases (Fig 2, 4, and 6). Negative deflections preceded larger $R$ waves
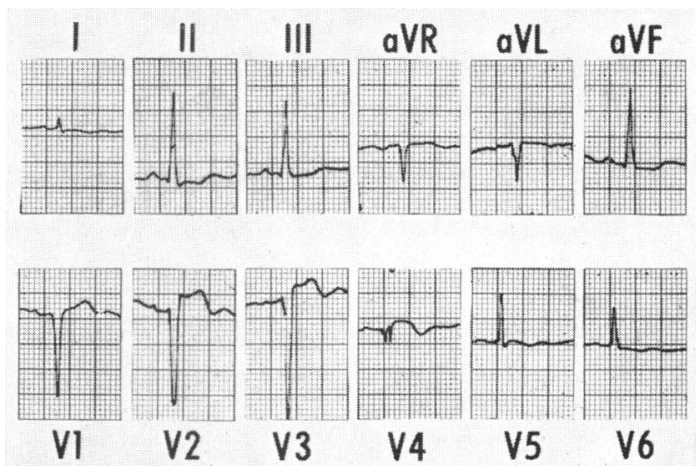

FIG. I (Case I) Sinus rhythm and acute anteroseptal myocardial infarction. 


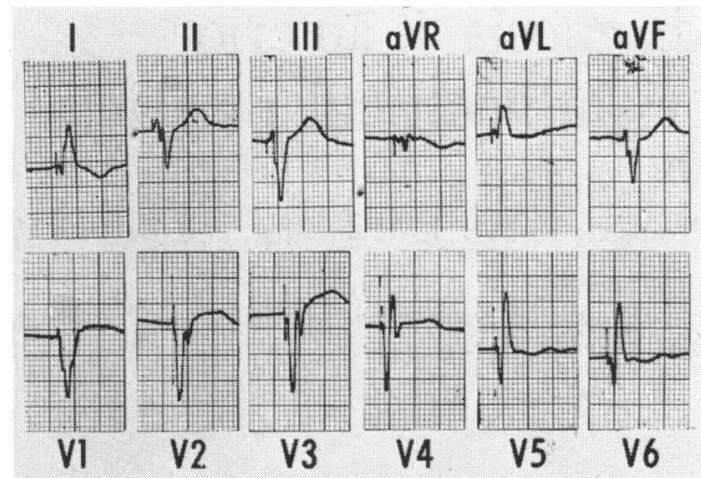

FIG. 2 (Case I) St-qR pattern during right ventricular apical (bipolar) pacing. Note the small negative deflections following the spike and preceding the larger $R$ waves in leads $I, a V L, V 4, V 5$, and V6. ST segment changes were not diagnostic of electrical injury since the electrocardiogram was obtained one week after the myocardial infarction. Note: St= stimulus artefact or spike which should not be confused with ST segment.

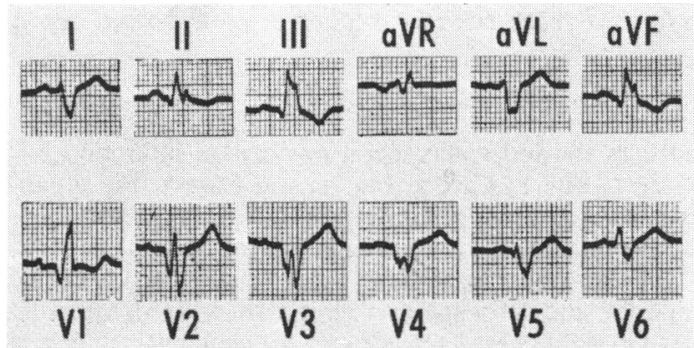

FIG. 3 (Case 2) Sinus rhythm with right bundlebranch block and left posterior hemiblock in a patient with acute anteroseptal myocardial infarction.

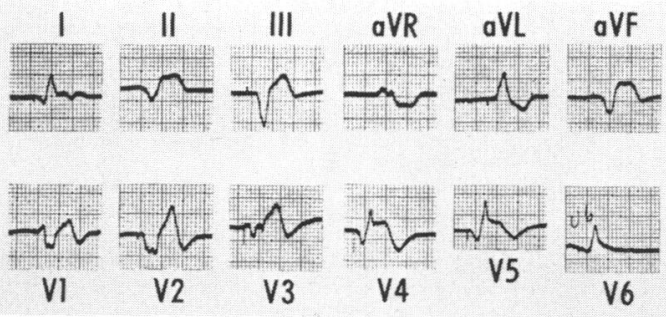

FIG. 4 (Case 2) Right ventricular apical (bipolar) pacing. The St-qR pattern is seen in leads $I, V_{3}, V_{4}$, and V5. Abnormal ST segment elevation follows the $S t-q R$ pattern in leads $V_{3}, V_{4}$, and $V_{5}$.
(St-qR pattern) in leads I, aVL, and (except for Case 3, Fig. 6) in lead V6 (Fig 2, 4, 6, and 8). Abnormal ST segment elevation following the St-qR pattern was observed only in Case 2 (Fig. 4 (leads $\mathrm{V}_{3}, \mathrm{~V}_{4}$, and $\mathrm{V}_{5}$ )) and Case 4 (not shown).

The outcome of the patients was as follows: Case I was alive six months after the myocardial infarction; Case 2 died three months later when he had a further infarction; Case 3 died while in the Coronary Care Unit due to cardiogenic shock; and Case 4 was discharged alive from the hospital with a permanent pacemaker.

\section{Discussion}

Sinus beats having a left bundle-branch block pattern with small $q$ waves in leads $I_{,}$aVL, V5, and V6

According to Sodi-Pallares et al. (1952, 1963), this association implies that left bundle-branch block is complicated by anteroseptal myocardial infarction. In the presence of advanced degrees of block (without myocardial infarction) the impulse emerging from the right bundle-branch initially propagates in a leftward, inferior, and slightly anterior direction. This general sequence of activation occurs because the wavefront depolarizing the right ventricular free wall is overpowered by the one which is simultaneously activating the electrically-predominant interventricular septum. Hence, an initial positive deflection will be recorded in leads I, V4, $\mathrm{V}_{5}, \mathrm{~V} 6, \mathrm{aVF}$, and (usually) V2.

If left bundle-branch block is associated with an extensive anteroseptal myocardial infarction extending close to the right septal areas first activated by the impulse emerging from the right bundlebranch, the latter cannot move towards the left (since these areas are infarcted). Hence, propagation can now occur, unopposed, throughout the right ventricular wall.

The initial electrical forces will point towards the right. They are responsible for the inscription of small q waves in leads I, aVF, $V_{4}, V_{5}$, and V6. This smaller deflection is followed by the larger, slurred, $R$ wave resulting from propagation in a leftward and posterior direction across the non-infarcted parts of the interventricular septum.

This need not be the only type of activation pattern in left bundle-branch block associated with septal myocardial infarction. For instance in other cases the initial vectors might point posteriorly and to the left resulting in a small q wave in a low placed V6 and in an R wave in leads I and aVL.

Primary ST-T abnormalities suggestive of myocardial infarction are present only during the acute phase. In later stages they are replaced by ST-T changes secondary to the left bundle-branch block (Sodi-Pallares et al., 1963; Massie and Walsh, 1960). 


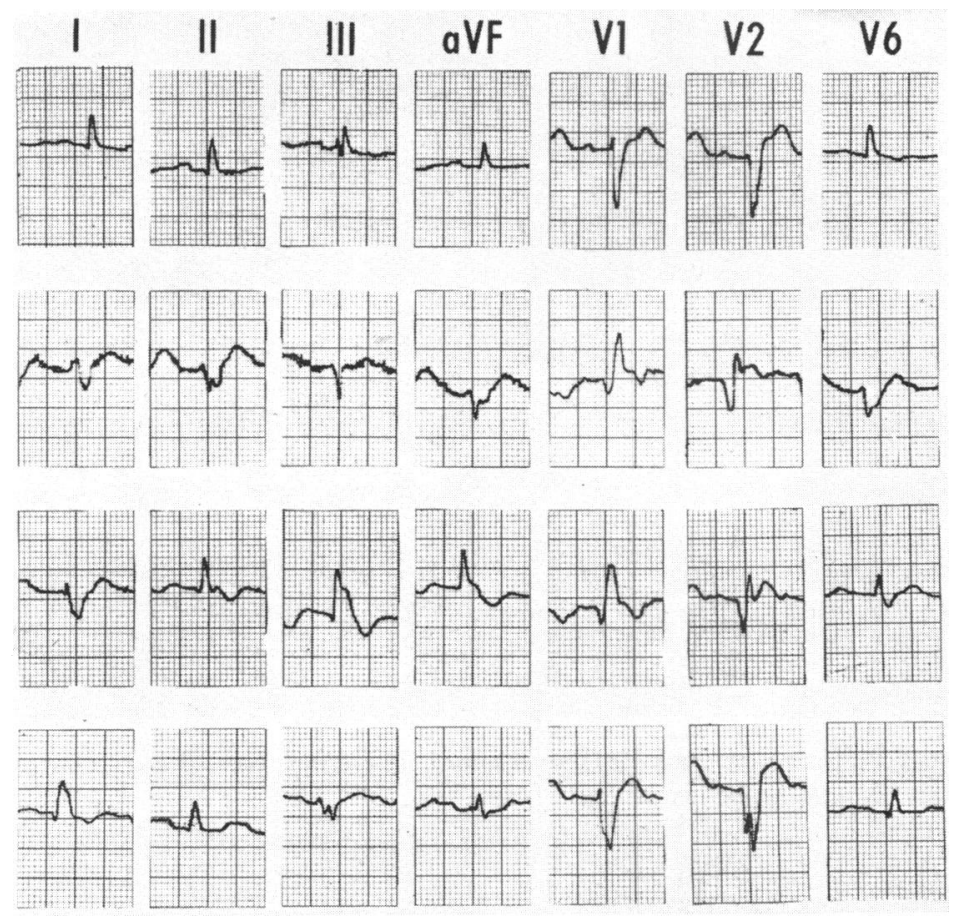

FIG. 5 (Case 3) Acute anterior wall myocardial infarction showing from top to bottom: control tracing with normal electrical axis; right bundle-branch block with left anterior hemiblock; right bundle-branch block with left posterior hemiblock; and left bundle-branch block with small $q$ waves in leads I and V6.

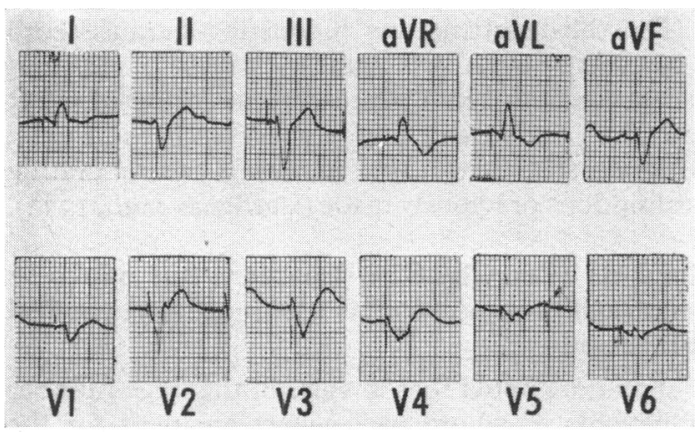

FIG. 6 (Case 3) Right ventricular apical (bipolar) pacing. The St-qR pattern is seen in leads I and aVL. Note the resemblance between these leads and the same ones in the bottom strip of Fig. 5, which were indicative of left bundle-branch block complicated by anteroseptal myocardial infarction.
Right ventricular paced beats with left bundlebranch block pattern and small, initial, negative deflections in leads $I, a V L, V_{5}$, and $V_{6}$ (St-qR pattern)

In the absence of anteroseptal myocardial infarction, beats resulting from stimulation of the right ventricular apex show a leftward and superior deviation of both initial vectors and electrical axis (Castellanos and Lemberg, 1969; Castellanos et al., 1970). This sequence of activation explains the slurred and predominantly positive deflections ( $R$ wave) in leads I and aVL. The morphology in the left chest leads ( $R, R s$, or $r S$ ) depends on the (horizontal) level at which the chest electrodes are placed (Castellanos and Lemberg, 1969).

An associated, extensive, anteroseptal myocardial infarction extending close to the site where the stimulating electrodes are located will change the direction of the initial QRS vectors. They will no longer point to the left, but to the right, because of the unopposed activation of the right ventricular wall. This type of propagation is responsible for the negative deflection seen between pacemaker stimulus 


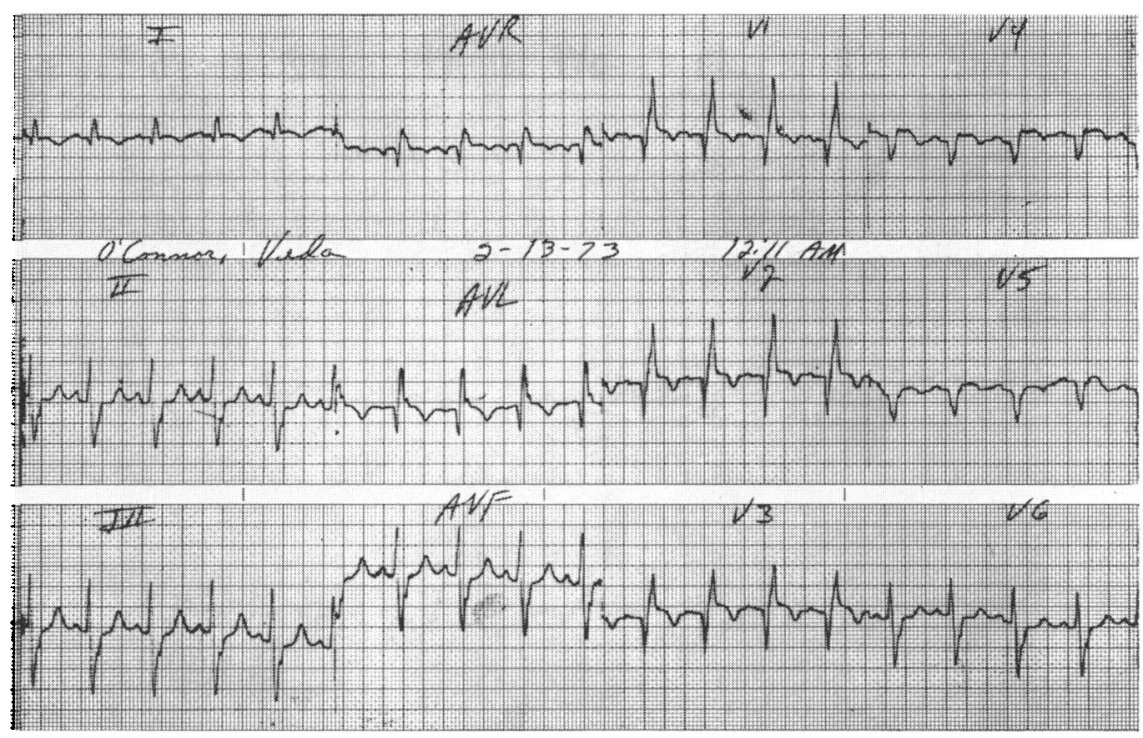

FIG. 7 (Case 4) Recent anterior wall myocardial infarction with $Q$ waves in leads $V_{I}-V_{5}$ and aVL. Right bundle-branch block was also present.
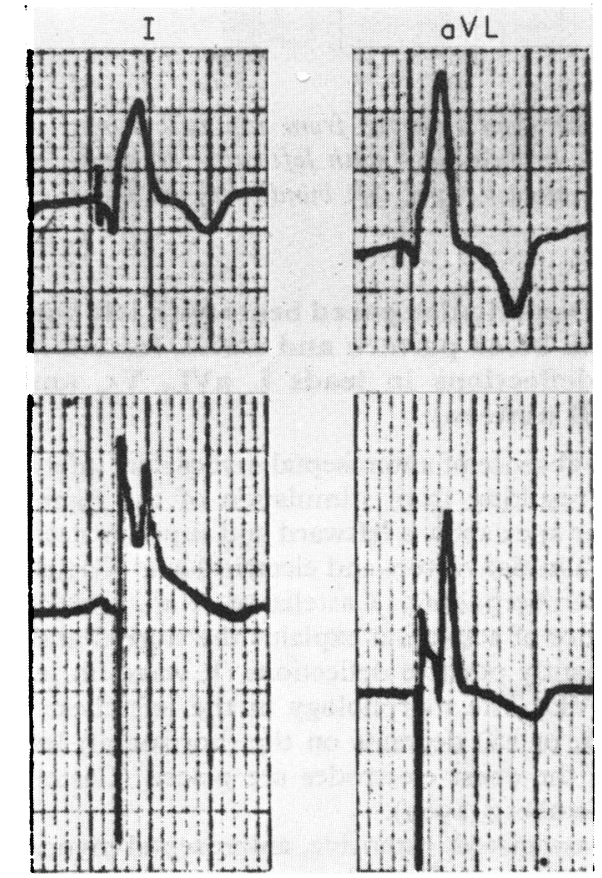

FIG. 8 (Case 4) Temporary right ventricular apical bipolar stimulation resulting in the St-qR pattern in leads $I$ and aVL (top). A permanent unipolar pacemaker was implanted because of persistent $A V$ block (bottom). Note that a (negative) deflection below the baseline did not occur during unipolar pacing. artefact and $R$ wave (St-qR pattern) in leads $I$ and aVL. However, the size of the positive deflection in the left chest leads depends on the horizontal level of electrode positioning. This explains why the morphology of the QRS complexes in $\mathrm{V}_{5}$ and V6 was different in Fig. 6 from that in Fig. 2 and 4. ST segment elevation following the St-qR pattern depends on the stage of the infarction in which the electrocardiogram is obtained. It can also be expected to occur in patients with ventricular aneurysms.

The third patient was interesting because both sinus beats (with left bundle-branch block) and pacemaker-induced QRS complexes showed a $\mathrm{qR}$ pattern in leads I and V6 (compare leads I in Fig. 5, bottom, and with 6), therefore corroborating assumptions previously made (Cardenas et al., I972).

\section{Reasons as to why St-qR pattern has not been previously recognized as a sign of myocardial infarction}

It should be noted that in spite of the large number of patients in whom pacemakers are used for the treatment of myocardial infarction, few authors had reported that the St-qR pattern indicated anterior wall myocardial infarction in patients with pacemakers (Cardenas et al., 1972; Zoneraich and Zoneraich, 1971).

Failure to observe these changes can be due in part to the use of unipolar stimulation, which is 
characterized by the large size of the spikes. The distortion that unipolar spikes can produce in the electrocardiogram might prevent determination as to whether the movement of the stylus between the spike and onset of QRS complex is an artefact produced in the electrocardiographic machine by the electrical impulse, or a 'true' reflection of an alteration in ventricular activation. In certain cases unipolar spikes can be large enough to distort the ST segment as well as the QRS complex. This creates pseudo-injury patterns which make the diagnosis of acute infarction difficult (Castellanos and Lemberg, 1969).

Even bipolar spikes can distort the baseline after delivery of the impulse if current intensity is high. Thus the mere fact that bipolar stimulation is used does not assure that the St-qR pattern will be recorded in patients with anterior myocardial infarction.

During right ventricular apical pacing the St-qR pattern will not be recorded when the infarction is limited to the left septal surface, since in these cases the initial forces can propagate in a leftward direction for a sufficient period of time so as to yield a positive deflection in leads $I, a V L$, and V6.

Moreover, even if an infarction were to involve the whole of the lower portions of the interventricular septum the St-qR pattern might not be seen if stimulation were to be performed, not from the right ventricular apex, but from a higher level (closer to the right ventricular outflow tract). In these cases the first septal muscle to be activated might not be infarcted at all. Hence, propagation can occur throughout the non-infarcted portions of the (high) septum in an inferior and leftward direction and produce an initial positive deflection in leads I, aVL, and left chest leads.

\section{References}

Cardenas, M., Sanz, G., Linares, J. C., Zamora, C., Medrano, G. A., and Estandia, A. (1972). Diagnostico electrocardiografico de infarto del miocardio en pacientes co estimulacion endocardica del ventriculo derecho por marcapasos. Archivos del Institute de Cardiologia de México, 42, 345.

Castellanos, A., Jr., and Lemberg, L. (I969). Electrophysiology of Pacing and Cardioversion. Appleton-Century-Crofts; New York. Butterworths, London.

Castellanos, A., Jr., Ortiz, J. M., Pastis, N., and Castillo, C. (1970). The electrocardiogram in patients with pacemakers. Progress in Cardiovascular Diseases, 13, 190.

Massie, E., and Walsh, T. J. (1960). Clinical Vectorcardiography and Electrocardiography, pp. 324-327. Year Book Publishers, Chicago.

Sodi-Pallares, D., Cisneros, F., Medrano, G. A., Bisteni, A., Testelli, M. R., and De Micheli, A. (1963). Electrocardiographic diagnosis of myocardial infarction in presence of bundle branch block (right and left), ventricular premature beats and Wolff-Parkinson-White syndrome. Progress in Cardiovascular Diseases, 6, 107.

Sodi-Pallares, D., Rodriquez, M. I., and Bisteni, A. (1952). Diagnostico electrocardiografico del bloqueo de rama izquierda complicado con infarto del miocardio. Archivos del Instituto de Cardiología de México, 22, 1 .

Zoneraich, O., and Zoneraich, S. (I97I). Pacemaker vectorcardiography in patients with myocardial infarction and intraventricular conduction defects. In Vectorcardiography, Vol. 2, p. 314. Hoffman. Ed. by I. North Holland Publishing Company, Amsterdam.

Requests for reprints to Dr. Agustin Castellanos, Jr., Section of Cardiology, Department of Medicine, School of Medicine, University of Miami, P.O. Box 875, Biscayne Annex, Miami, Florida 33152, U.S.A. 